\title{
CORRECTIONS
}

\section{The hospital bed: on its way out?}

We made a mistake in the numbering of the y axis of figure 3 in this Data Briefing article by John Appleby (BMJ

2013;346:f1563, doi:10.1136/bmj.f1563). The top three numbers ๑ BMJ Publishing Group Ltd 2013 (upwards) should be 85, 90, 95 [not 95, 80, 95 as published]. 